\title{
Clinicopathologic study of small hepatocellular carcinoma with microscopic satellite nodules to determine the extent of tumor ablation by local therapy
}

\author{
KOZO IKEDA $^{1}{ }^{1}$, TOSHIHITO SEKI ${ }^{1}$, HIDETO UMEHARA ${ }^{1}$, RYOSUKE INOKUCHI $^{1}$, TORU TAMAI ${ }^{1}$, \\ NORIKO SAKAIDA $^{2}$, YOSHIKO UEMURA ${ }^{2}$, YASUO KAMIYAMA ${ }^{3}$ and KAZUICHI OKAZAKI ${ }^{1}$ \\ Departments of ${ }^{1}$ Gastroenterology and Hepatology, ${ }^{2}$ Clinical Pathology, and ${ }^{3}$ Surgery, \\ Kansai Medical University, 10-15 Fumizono-cyo, Moriguchi, Osaka, Japan
}

Received March 26, 2007; Accepted May 25, 2007

\begin{abstract}
To determine the optimal treatment margin of local ablation therapy for small hepatocellular carcinoma (HCC), we investigated characteristics of microscopic satellite HCC nodules (msn) using resected livers, and the incidence of local recurrences in patients who underwent percutaneous microwave coagulation therapy (PMCT) according to whether or not an adequate treatment margin was achieved. We reviewed 117 single small HCCs (tumor size $\leq 3 \mathrm{~cm}$ ) resected with a $\geq 1-\mathrm{cm}$ surgical margin. Among the surgically resected tumors, none of the msn were detected by preoperative imaging. When an msn was observed in the resected specimen, the maximum distance from the edge of the tumor to the msn was measured. Among the tumors sized $\leq 2 \mathrm{~cm}(\mathrm{n}=66)$, the number having msn and the distance $(\mathrm{mm})$ from the main tumor according to the degree of tumor differentiation were as follows: well $(n=16), 1(6.2 \%)$ and $1.4 \mathrm{~mm}$; moderate $(n=46), 5(19.2 \%)$ and $5.8 \pm 1.2 \mathrm{~mm}$; and poor $(\mathrm{n}=4), 1(25.0 \%)$ and $4.8 \mathrm{~mm}$. Among the tumors sized 2 to $3 \mathrm{~cm}(\mathrm{n}=51)$, the corresponding results were: well $(n=11), 3(27.2 \%)$ and $3.5 \pm 2.2 \mathrm{~mm}$; moderate $(\mathrm{n}=36), 6(16.7 \%)$ and $5.4 \pm 1.4 \mathrm{~mm}$; and poor $(\mathrm{n}=4), 1(25.0 \%)$ and $4.9 \mathrm{~mm}$. Of the 112 vascular tumors, 17 (15\%) had msn. The 5 avascular tumors had no msn. Among the patients who underwent PMCT with a treatment margin measuring $<5 \mathrm{~mm}$, local recurrence occurred in 2 of 25 patients whose original tumor was $\leq 2 \mathrm{~cm}$, and 3 of $25(12 \%)$ patients of those with tumors of 2 to $3 \mathrm{~cm}$. No patient with a tumor $\leq 3 \mathrm{~cm}$ and a $\geq 5$-mm treatment margin suffered a local recurrence. The results of this study suggest that the incidence of local recurrence may be reduced by achieving a treatment margin of any width for avascular tumors and a margin of $1 \mathrm{~cm}$ for
\end{abstract}

Correspondence to: Dr Kozo Ikeda, Department of Gastroenterology and Hepatology, Kansai Medical University, 10-15 Fumizono-cho, Moriguchi, Osaka 570-8507, Japan

E-mail: ikedakoz@takii.kmu.ac.jp

Key words: hepatocellular carcinoma, local ablation therapy, treatment margin vascular tumors during local ablation therapy for small HCCs of $\leq 3 \mathrm{~cm}$.

\section{Introduction}

Local ablation therapy for hepatocellular carcinoma (HCC) has been performed by three common techniques; ethanol injection therapy (EIT) based on the dehydration and fixation effects of ethanol $(1,2)$, microwave coagulation therapy (MCT) based on dielectric heat generated by microwaves (3-5), and radiofrequency ablation (RFA) based on the Joules of heat generated by radiofrequency waves (6-8). The aim of percutaneous local ablation therapy is to achieve complete necrosis of the target tumor. However, recurrences can occur near or adjacent to the treated lesion even when the therapy causes complete necrosis on imaging studies (9-11). In the case of HCC, it is well known that microscopic satellite nodules (msn) exist near the tumor, although imaging modalities usually are unable to detect such nodules (12-14). Even though msn that cannot be detected by imaging are not the targets of local ablation therapy, these lesions may be an important risk factor for local recurrences. Therefore, we should expand the range of necrosis so that it includes not only the tumor but also the msn (i.e., those that cannot be detected by imaging) to reduce the incidence of local recurrences. In other words, more widespread necrosis should be achieved around the border or capsule of the tumor. However, many HCC patients have cirrhosis as their background liver disease, raising the possibility that excessively extensive necrotic damage to the hepatic parenchyma could lead to a poor prognosis (15-18).

To decide how large a necrotic area is needed to include most msn, we histologically investigated the characteristics of these nodules, such as their frequency, size, and distance from the tumor. For this study, we need to introduce the concept of a 'treatment margin' for local ablation therapy of HCC, which corresponds to the 'surgical margin' used for tumor resection (19-22). However, few studies on the treatment margin for the local ablation of HCC have been published. In this study, we used resected specimens to measure the distance from the tumor to the msn, and clinicopathologically investigated how extensive the range of necrosis should be to achieve local control by percutaneous therapy. 
Table I. Clinical features of small hepatocellular carcinomas.

\begin{tabular}{lccc}
\hline & Tumor size $\leq 2 \mathrm{~cm}(\mathrm{n}=66)$ & Tumor size $>2$ but $\leq 3 \mathrm{~cm}(\mathrm{n}=51)$ & p-value \\
\hline Average size $(\mathrm{cm})$ & $1.7(1.5 \pm 0.5)$ & $2.6(2.6 \pm 0.5)$ & $<0.005$ \\
Gender (M:F) & $50: 13$ & $45: 7$ & 0.161 \\
Age (years) & 67.1 & 65.4 & 0.837 \\
AST (IU/1) & $72.9(138 \pm 116)$ & $72.5(106.5 \pm 84.5)$ & 0.453 \\
ALT (IU/1) & $69.1(119.5 \pm 105.5)$ & $76.9(120 \pm 106)$ & 0.495 \\
T-Bil (mg/dl) & $0.7(1.0 \pm 0.7)$ & $0.9(1.1 \pm 0.8)$ & 0.205 \\
PT (\%) & $87.0(71 \pm 42)$ & $90.0(91.5 \pm 28.5)$ & 0.937 \\
Pit & $11.9(17.4 \pm 12.6)$ & $12.7(17.5 \pm 12.8)$ & 0.770 \\
Alb (g/dl) & $3.9(4.1 \pm 0.5)$ & $3.7(3.7 \pm 0.5)$ & 0.521 \\
ICGR15(\%) & $17.3(17.05 \pm 8.75)$ & $132.5(16.6 \pm 11.3)$ & 0.479 \\
AFP (ng/ml) & $168.9(649.6 \pm 647.2)$ & $33.1(41.5 \pm 41.5)$ & 0.433 \\
AFP-L3 (\%) & $25.2(34.2 \pm 29.8)$ & $468.1(2288.5 \pm 2285.5)$ & 0.652 \\
PIVKAII (mAU/ml) & $293.5(795 \pm 792)$ & $47: 4: 0$ & \\
Child-Pugh (A:B:C) & $62: 4: 0$ & & 0.919 \\
Etiology & & 39 & 0.400 \\
HCV & 51 & 9 & 0.154 \\
HBV & 8 & 0 & 0.719 \\
Alcohol & 2 & 3 & \\
Unknown & 5 & & \\
\hline
\end{tabular}

AST, serum aspartate aminotransferase level; ALT, serum alanine aminotransferase level; T-Bil, serum total bilirubin level; Alb, serum albumin level; PT, prothrombin time; Pit, platelete count; ICGR15, iodocyanine green retention at 15 min; AFP, serum $\alpha$-fetoprotein level; AFP-L3, Lens culinaris agglutinin A-reactive fraction of $\alpha$-fetoprotein; PIVKAII, protein-induced vitamin K antagonist absence II; HCV, hepatitis $\mathrm{C}$ virus; $\mathrm{HBV}$, hepatitis B virus.

Additionally, the incidence of local recurrence was evaluated in patients who underwent percutaneous microwave coagulation therapy (PMCT) according to whether or not an adequate treatment margin was achieved.

\section{Materials and methods}

Subjects. From January 1995 to December 2005, 117 patients with a single solitary HCC measuring $\leq 3 \mathrm{~cm}$ in maximum diameter were admitted to our institution and underwent tumor resection with a surgical margin of $\geq 1 \mathrm{~cm}$. The preoperative clinical features of these 117 patients are shown in Table I. All patients were diagnosed as having $\mathrm{HCC}$ by pathologic examination after surgery. Tumor nodules that showed a tumor stain on a preoperative computed tomographic angiography (CT-A) were defined as vascular HCC. Nodules that were isodense or had a low density and were not stained on CT-A were defined as avascular HCC. Avascular HCCs were subjected to a preoperative ultrasound (US) guided tumor biopsy for the histopathologic confirmation of the diagnosis of HCC. The tumor was seen as a perfusion defect on arterioportography (CT-AP) in all cases.

Another 116 patients with a solitary HCC measuring $\leq 3 \mathrm{~cm}$ in maximum diameter who underwent PMCT between January 1997 and December 2002 at our institution were enrolled in this study. There were 69 patients with tumors measuring $\leq 2 \mathrm{~cm}$, and 47 patients with tumors measuring $>2 \mathrm{~cm}$ but $\leq 3 \mathrm{~cm}$ in maximum diameter. A tumor stain was observed in all of these patients. All of these patients underwent transcatheter hepatic arterial chemoembolization (TACE) with iodized oil (4 to $6 \mathrm{ml}$ of Lipiodol; Andre Guerbet, Anlnay-sousBios, France) before PMCT, and showed uniform accumulation of this oil in the target tumor.

Written informed consent was obtained from each patient before every treatment.

Evaluation of the frequency of microscopic satellite nodules (msn) and their distance from the tumor. A lesion was defined as a microscopic satellite nodule when it could not be detected by any imaging technique, but could be confirmed by microscopy. In addition, it was small and located close to the primary tumor, and showed the same histology as the primary tumor or was poorly differentiated. Resected tumor specimens were cut along their maximum diameter and fixed in $10 \%$ formalin. After $24 \mathrm{~h}$, the specimens were cut into 5-mm thick slices and it was confirmed that no other tumors existed in the noncancerous areas of the specimens that were visible to the naked eye. Next, each specimen was embedded in paraffin, cut into $2-\mu \mathrm{m}$ sections using a microtome, and then stained with hematoxylin and eosin. We reviewed all of the histologic slides to evaluate the grade of tumor differentiation, the fibrous capsule, and capsular infiltration. If the tumor had msn, the maximum distance from the tumor to these lesions and the maximum diameter of the msn were measured on the histologic slides using a micrometer (Fig. 1). The histologic grade of the tumor was diagnosed from the predominant grade among 


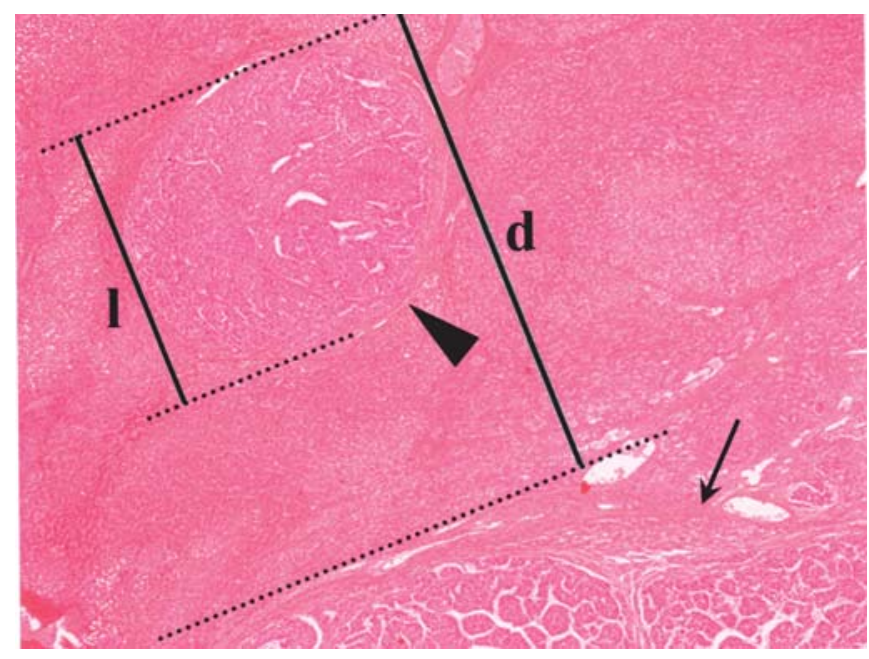

Figure 1. Measurement of the maximum distance (d) between the tumor (arrow) and the msn (arrowhead), and the maximum diameter of the msn (1).

the cancer cells occupying one nodule. If the tumor had multiple msn, the longest distance between the tumor and the msn was measured. The incidence of msn was investigated in relation to the tumor size, vascularity, and histologic grade of the tumor.

PMCT technique and evaluation of therapeutic efficacy. All patients underwent TACE with Lipiodol before PMCT, which was performed within 7 days of TACE. PMCT was carried out as described previously $(3,4)$. After local anesthesia was provided, a 13-gauge guide needle was inserted near the tumor under ultrasonic guidance. After the inner stylet of the guide needle was removed, the microwave electrode was inserted through the outer part of the needle until it reached the tumor. Next, the electrode was connected to the microwave generator via a flexible coaxial cable and the tumor zone was irradiated with microwaves. Subsequently, the electrode and the outer needle were removed. The needle track also was irradiated to prevent bleeding from the liver surface after the electrode was removed. During each PMCT session, multiple electrode insertions were performed for each tumor at different sites, and microwave irradiation ( $80 \mathrm{~W}$ for $5 \mathrm{~min}$ ) was delivered at each site of insertion until an extensive hyperechoic change was observed covering a zone that not only included the tumor, but also the adjacent noncancerous tissue. When this change was seen on ultrasound, the initial session was complete. The maximum number of electrode insertions was 4 for each session. To assess the zone of necrosis, dynamic CT was performed 2 to 3 days after each treatment. The endpoint of treatment was defined as an attenuation of the lesion (including the surrounding liver parenchyma) on dynamic CT after therapy. Patients received additional PMCT sessions until the treatment was judged to be complete. All patients were closely followed by ultrasonography, dynamic CT, or dynamic MRI, performed every 3 to 4 months after discharge.

Measurement of the treatment margin and evaluation of local recurrence according to the margin. The treatment margin was defined as the shortest distance measured from the external border of the accumulated iodized oil to the outer border of
Table II. Pathomorphologic features and vascularity of HCCs.

\begin{tabular}{lccc}
\hline $\begin{array}{l}\text { Tumor } \\
\text { differentiation }\end{array}$ & $\begin{array}{c}\text { Tumor size } \\
\leq 2 \mathrm{~cm}(\mathrm{n}=66)\end{array}$ & $\begin{array}{c}\text { Tumor size }>2 \text { but } \\
\leq 3 \mathrm{~cm}(\mathrm{n}=51)\end{array}$ & $\mathrm{p}$-value \\
\hline Well & 16 & 11 & \\
fc $(+)$ & 11 & 8 & 0.836 \\
fc-inf (+) & 3 & 6 & 0.128 \\
msn (+) & 1 & 3 & 0.337 \\
Vascularity (+) & 14 & 9 & 0.886 \\
Moderate & 46 & 36 & \\
fc (+) & 38 & 31 & 0.899 \\
fc-inf (+) & 28 & 27 & 0.256 \\
msn (+) & 5 & 6 & 0.872 \\
Vascularity (+) & 45 & 36 & 0.930 \\
Poor & 4 & 4 & \\
fc (+) & 4 & 1 & 0.144 \\
fc-inf (+) & 3 & 1 & 0.480 \\
msn (+) & 1 & 1 & 0.414 \\
Vascularity (+) & 4 & 4 & \\
\hline
\end{tabular}

fc, fibrous capsule; fc-inf, capsular infiltration; msn, microscopic satellite nodule.

the unstained region of hypoattenuation on axial dynamic CT scans obtained after treatment. If the tumor was adjacent to large vessels or close to the liver surface, the treatment margin was defined as the shortest distance that could be measured to the hepatic parenchyma itself. Local recurrence was defined as a recurrent tumor that was located at, or adjacent to, the region of hypoattenuation on dynamic CT. We divided the subjects into two groups, those who had a treatment margin of $<5 \mathrm{~mm}$ or $\geq 5 \mathrm{~mm}$, and then retrospectively investigated the incidences of local recurrence at 3 years after therapy.

Statistical analysis. Patient background factors, the size of the msn, and the distance between the tumor and msn were compared by Student's t-test after stratification according to the maximum diameter of the tumor. We used the chi-square test to compare the frequency of each histologic type and the frequency of msn, applying Yates's correction when needed. A p-value $<0.05$ was considered statistically significant.

\section{Results}

Pathology and vascularity of the primary tumors. Among the patients with tumors measuring $\leq 2 \mathrm{~cm}$, capsular invasion and msn were observed in 34 and 7 patients, respectively. On the other hand, among the patients with tumors measuring $>2 \mathrm{~cm}$ but $\leq 3 \mathrm{~cm}$, capsular invasion and msn were observed in 34 and 10 patients, respectively. There was no difference in the frequency of tumor vascularity according to primary tumor size or degree of differentiation (Table II).

Frequency of msn in relation to tumor diameter and vascularity. Among the patients with vascular tumors, msn were 
Table III. Number of cases with msn stratified according to tumor size, tumor differentiation and tumor vascularity.

\begin{tabular}{|c|c|c|c|c|c|c|}
\hline \multirow{3}{*}{ Vascularity (+) } & \multicolumn{3}{|c|}{$\begin{array}{l}\text { Tumor size } \\
\leq 2 \mathrm{~cm}\end{array}$} & \multicolumn{3}{|c|}{$\begin{array}{l}\text { Tumor size } \\
>2 \text { but } \leq 3 \mathrm{~cm}\end{array}$} \\
\hline & & Well & 1 & & Well & 3 \\
\hline & 7 & Moderate & 5 & 10 & Moderate & 6 \\
\hline & & Poor & 1 & & Poor & 1 \\
\hline
\end{tabular}

Vascularity (-) $\quad 0$

0

Tumor size $\leq 2 \mathrm{~cm}$ vs. tumor size $>2$ but $\leq 3 \mathrm{~cm}$ : $\mathrm{p}=0.174$.

Table IV. Maximum diameter of msn (mm) according to tumor size and tumor differentiation (mean $\pm \mathrm{SD})$.

\begin{tabular}{lrrr}
\hline $\begin{array}{l}\text { Tumor } \\
\text { differentiation }\end{array}$ & $\begin{array}{c}\text { Tumor size } \\
<2 \mathrm{~cm}\end{array}$ & $\begin{array}{r}\text { Tumor size } \\
>2 \text { but } \leq 3 \mathrm{~cm}\end{array}$ & p-value \\
\hline Well & $1.8(\mathrm{n}=1)$ & $3.7 \pm 2.8(\mathrm{n}=3)$ & 0.500 \\
Moderate & $4.4 \pm 1.2(\mathrm{n}=5)$ & $3.8 \pm 2.6(\mathrm{n}=6)$ & 0.268 \\
Poor & $5.0(\mathrm{n}=1)$ & $3.9(\mathrm{n}=1)$ & \\
\hline
\end{tabular}

detected in 7 patients who had tumors measuring $\leq 2 \mathrm{~cm}$ in diameter and in 10 patients who had tumors measuring $>2 \mathrm{~cm}$ in diameter, and there was no difference in the frequency of msn between these groups. No msn were detected in the patients with avascular tumors, irrespective of tumor diameter. None of the nodules surrounding the primary tumors showed a higher level of differentiation than did the tumors themselves (Table III).

Size of msn in relation to tumor diameter and differentiation. The size of the msn was $1.8 \mathrm{~mm}$ in the one patient who had a well-differentiated $\mathrm{HCC}$ of $\leq 2 \mathrm{~cm}$, while it was $4.4 \pm 1.2 \mathrm{~mm}$ (mean \pm SD) in those $(n=5)$ with moderately differentiated $\mathrm{HCC}$ and $5 \mathrm{~mm}$ in the patient with a poorly differentiated HCC of the same size. Among the patients with a tumor measuring $>2 \mathrm{~cm}$, the sizes of the msn were $3.7 \pm 2.8,4.5 \pm 3.4$, and $3.9 \mathrm{~mm}$ for well, moderately, and poorly differentiated tumors, respectively (Table IV).

Distance between vascular primary tumors and msn. The mean distance between the primary tumor and the msn was $5.3 \pm 2.1 \mathrm{~mm}$ (maximum: $8.4 \mathrm{~mm}$ ) in patients who had tumors measuring $\leq 2 \mathrm{~cm}$ in diameter, while it was $4.8 \pm 1.9 \mathrm{~mm}$ (maximum: $7.6 \mathrm{~mm}$ ) in those who had tumors measuring $>2 \mathrm{~cm}$ in diameter. This difference was not statistically significant (Fig. 2).

Local recurrence after PMCT. When the treatment margin was $<5 \mathrm{~mm}$, local recurrences occurred in 2 of the $25(8 \%)$ patients who had a tumor $\leq 2 \mathrm{~cm}$ in diameter and in 3 of the $25(12 \%)$ patients with a tumor $>2 \mathrm{~cm}$ in diameter (Fig. 3).

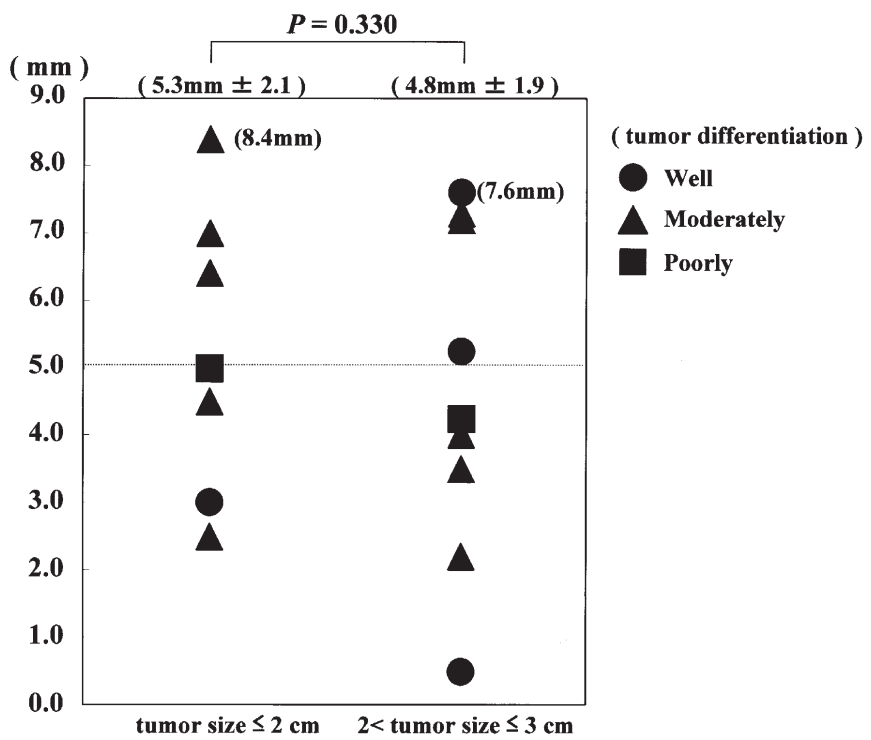

Figure 2. Maximum distance between vascular tumors and msn stratified according to tumor size.

When the treatment margin was $\geq 5 \mathrm{~mm}$, none of the patients with a tumor $\leq 3 \mathrm{~cm}$ in diameter suffered a local recurrence (Fig. 4, Table V). A treatment margin of at least $1 \mathrm{~cm}$ could be obtained in 12 patients $(17 \%)$ with a tumor $\leq 2 \mathrm{~cm}$ in diameter and in 6 patients $(13 \%)$ with a tumor measuring $>2 \mathrm{~cm}$ but $\leq 3 \mathrm{~cm}$ in diameter.

\section{Discussion}

At many institutions, 'minimally invasive loco-regional therapy', for HCC is performed by ethanol injection, microwave coagulation, or radiofrequency ablation under percutaneous ultrasonic guidance, laparoscopic guidance, or at open surgery, and there have been many reports referring to its usefulness $(2,4,8,23,24)$. However, when we perform local ablation therapy, the optimal extent of therapy to perform the desired therapeutic effect is often unclear. Generally, previous investigators have defined the end-point of therapy as uniform hypoattenuation on dynamic CT that covers the area of the tumor seen on dynamic CT before therapy $(2,8)$. However, this criterion does not allow for a margin such as that obtained with a surgical resection. In fact, there are no standards for such a margin in the case of local ablation therapy for HCC. In the case of surgical resection, the adequate surgical margin is still controversial. Generally, $\mathrm{a} \geq 1-\mathrm{cm}$ surgical margin or $\geq 2 \mathrm{~cm}$ is recommended to obtain a reasonable cumulative survival of patients with an HCC of $\leq 2 \mathrm{~cm}$ in diameter $(21,22)$. If a wide surgical margin is achieved, the possibility of a local recurrence is low, and the probability of obtaining a satisfactory survival rate is high. On the other hand, among patients treated with local ablation therapy, hepatic functional reserve is poorer than in those who undergo a surgical resection. Therefore, to obtain good local control without damaging any more hepatic parenchyma than is necessary, it is important to determine the adequate treatment margin for local ablation therapy.

Some reports related to local ablation therapy describe the termination of therapy after achieving an adequate margin, or 

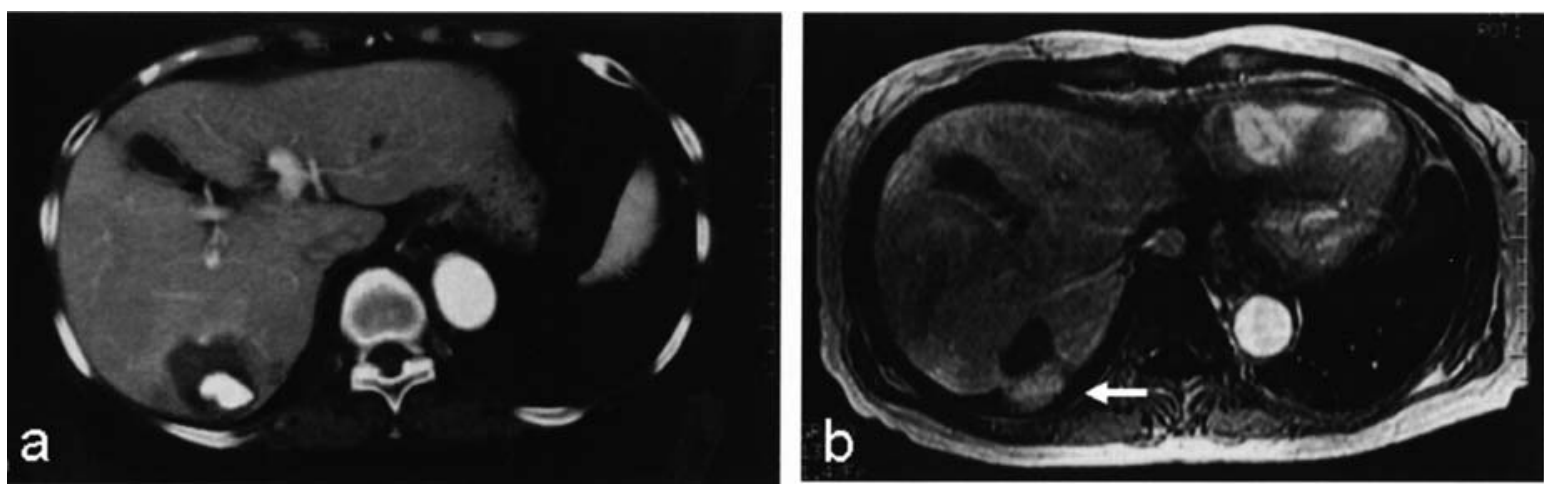

Figure 3. Dynamic CT and MRI following TACE and PMCT for a representative HCC measuring $2 \mathrm{~cm}$ in maximum diameter. (a) Three days after completion of treatment (dynamic CT, early phase). A treatment margin of $\geq 5 \mathrm{~mm}$ could not be obtained. (b) Six months later (dynamic MRI, early phase). Local recurrence is detected at the edge of the treated zone (white arrow).
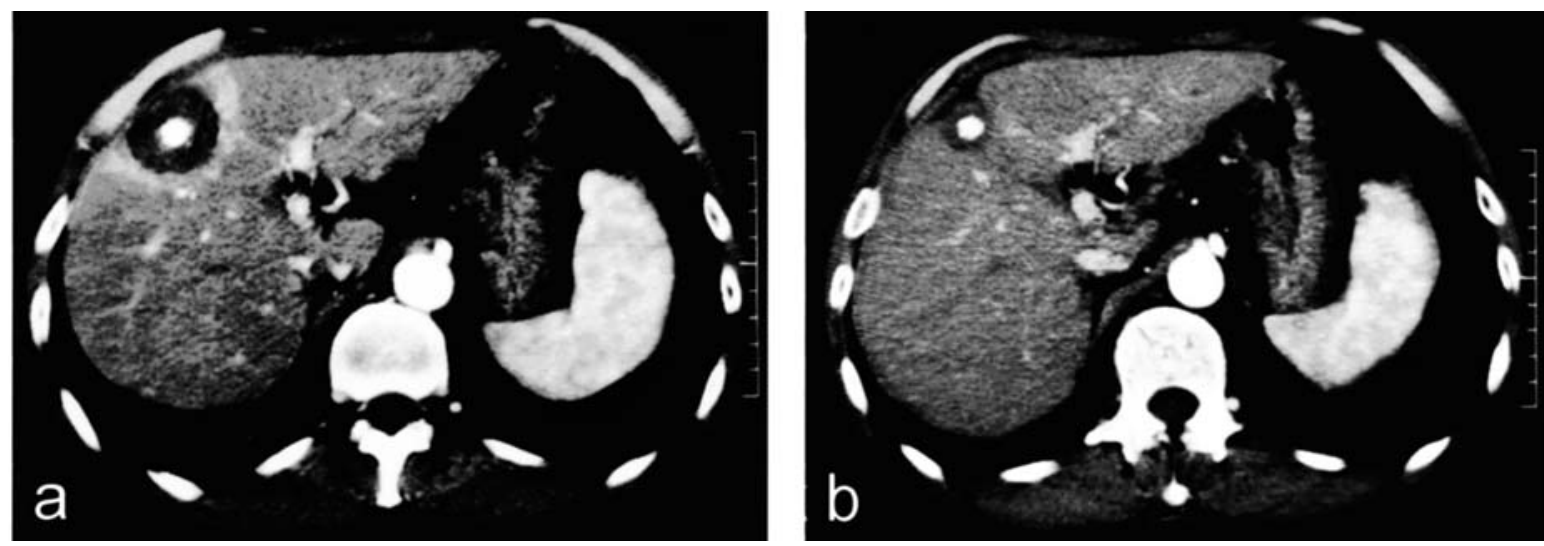

Figure 4. Dynamic CT following TACE and PMCT for a representative $\mathrm{HCC}$ of $1.5 \mathrm{~cm}$ in maximum diameter. (a) Three days after the completion of treatment (dynamic CT, late phase). Accumulation of iodized oil was observed in the tumor. TACE and PMCT achieved an adequate treatment margin (1 cm). (b) Three years later (dynamic CT, late phase). The treated regions show areas of shrinkage without local recurrence.

Table V. Number of cases showing local recurrence within 3 years after PMCT.

\begin{tabular}{lcc}
\hline Tumor size & Margin $\geq 5 \mathrm{~mm}$ & Margin $<5 \mathrm{~mm}$ \\
\hline$\leq 2 \mathrm{~cm}(\mathrm{n}=69)$ & $0 / 44$ & $2 / 25$ \\
$>2$ but $\leq 3 \mathrm{~cm}(\mathrm{n}=47)$ & $0 / 22$ & $3 / 25$ \\
\hline
\end{tabular}

in the case of local recurrences after therapy, they state that an adequate margin was not achieved. Nevertheless, it seems that most authors do not precisely define the margin they require to achieve the complete necrosis of the tumor $(8,11)$. Without objective criteria, the assessment of therapeutic efficacy becomes unclear and the comparison of results between studies may be difficult. Therefore, the end-point of therapy should be clearly defined in terms of the treatment margin. In our study, capsular invasion was frequently observed among small HCC of $\leq 3 \mathrm{~cm}$ in maximum diameter, so the necessity of achieving a margin is unquestionable. Furthermore, the presence of msn, which cannot be detected by various imaging modalities and are often found around the tumor, cannot be ignored, when local ablation therapy aims at achieving good local control comparable to that obtained with surgical resection (12-14). However, the identification of msn measuring $<5 \mathrm{~mm}$ in maximum diameter was judged to be difficult even with the most recent imaging techniques $(25,26)$. Such msn would be simultaneously removed with the tumor by surgical resection, even if they were not recognized on imaging, but there is a strong possibility of these lesions remaining untreated by local ablation therapy. Thus, the issue is how wide a margin should be to prevent local recurrence.

In our study, irrespective of the histologic grade of the tumor, msn were not seen in patients with avascular tumors measuring $\leq 3 \mathrm{~cm}$ in maximum diameter. Therefore, an unstained low-density area overlapping the tumor on dynamic $\mathrm{CT}$, or the presence of a treatment margin, could be considered to indicate that the therapy has been adequate and thus can be terminated in patients receiving local treatment for avascular tumors of $\leq 3 \mathrm{~cm}$ in diameter. In other words, if a treatment margin is obtained, necrosis of the tumor and any sites of capsular invasion can be achieved and adequate local control might be expected.

On the other hand, if the tumor is vascular, there is a strong possibility that msn will remain untreated irrespective 
of the histologic grade of the tumor. Among the tumors of $\leq 2 \mathrm{~cm}$, the incidence of msn was $11 \%(7 / 63)$ and the mean distance from the edge of the tumor to these lesions was $5.3 \mathrm{~mm}$ (maximum: $8.5 \mathrm{~mm}$ ). Among the tumors of $>2 \mathrm{~cm}$, the frequency of msn was $20 \%$ (10/49) and the mean distance from the tumor to these lesions was $4.8 \mathrm{~mm}$ (maximum: $7.5 \mathrm{~mm}$ ). Although msn occurred in patients with tumors of $>2 \mathrm{~cm}$ in diameter at approximately twice the frequency of those with tumors of $\leq 2 \mathrm{~cm}$ in diameter, the maximum distance of the msn from the main lesion was comparable between the two groups. For vascular tumors of $\leq 3 \mathrm{~cm}$ in maximum diameter, the distribution of the msn suggests that a $1-\mathrm{cm}$ margin may be required to achieve local good control similar to that after surgical resection. Okusaka et al (12) histopathologically investigated resected $\mathrm{HCC}$ that were $\leq 3 \mathrm{~cm}$ in maximum diameter, and reported that the satellite lesions of well/moderately differentiated HCC were located $\leq 1 \mathrm{~cm}$ from the tumor. Maeda et al (13) reported that the treatment margin of local ablation therapy should be at least $1 \mathrm{~cm}$ from the tumor especially in cases of moderate to poorly differentiated HCCs of $\leq 2 \mathrm{~cm}$ in maximum diameter. Our findings were similar to these reports concerning the treatment margin that should be achieved, although Maeda et al proposed margins that varied according to the histologic differentiation of the tumor. A margin should be established in each patient according to various factors, including the hepatic functional reserve, tumor size, tumor differentiation, and tumor location. However, it is uncommon to perform a tumor biopsy to examine the histologic differentiation in patients with vascular tumors, since these patients generally are diagnosed as having HCC by imaging techniques and/or tumor markers and then receive local ablation therapy. Therefore, in patients with HCC measuring $\leq 3 \mathrm{~cm}$ in maximum diameter, the treatment margin should be estimated from the tumor vascularity.

In this retrospective study of therapeutic results of PMCT, local recurrences occurred with a treatment margin of $<5 \mathrm{~mm}$, but not in vascular HCC tumors of $\leq 3 \mathrm{~cm}$ and with a margin of $\geq 5 \mathrm{~mm}$. Because the mean distance from the primary tumor to the msn was $5 \mathrm{~mm}$, it may be possible to achieve local control of tumors in many patients when a treatment margin of $\geq 5 \mathrm{~mm}$ is achieved. To ensure that vascular tumors measuring $\leq 3 \mathrm{~cm}$ in diameter are controlled, it is considered appropriate to aim for a margin of $1 \mathrm{~cm}$ based on the present pathologic results. However, as the diameter of a tumor increases, it becomes more difficult to achieve a $10-\mathrm{mm}$ necrotic margin. As a wider margin is obtained, the possibility of complications also will increase (15-18). In addition, considering the underlying liver diseases of patients who receive local ablation therapy, destroying the non-cancerous tissue around the target tumor may lead to a significant deterioration of liver function. Accordingly, among vascular tumors measuring $\leq 3 \mathrm{~cm}$ in maximum diameter, the decision regarding whether the margin should be more than $5 \mathrm{~mm}$ or $10 \mathrm{~mm}$ should not be based only on our study. Whether a treatment margin of $1 \mathrm{~cm}$ reduces local recurrences and improves the prognosis of these patients without significant deterioration of their liver function remains open to further investigation and prospective studies.

The results of this study suggest that the incidence of local recurrence may be reduced by aiming to achieve a treatment margin of any width for avascular tumors and a margin of at least $1 \mathrm{~cm}$ for vascular tumors during local ablation therapy for small $\mathrm{HCC}$ measuring $\leq 3 \mathrm{~cm}$ in diameter.

\section{References}

1. Ebara M, Ohto M, Sugiura N, et al: Percutaneous ethanol injection for the treatment of small hepatocellular carcinoma: study of 95 patients. J Gastroenterol 5: 616-626, 1990.

2. Ebara M, Okabe S, Kita K, et al: Percutaneous ethanol injection for small hepatocellular carcinoma: Therapeutic efficacy based on 20-year observation. J Hepatol 43: 458-464, 2005.

3. Seki T, Wakabayashi M, Nakagawa T, et al: Ultrasonically guided percutaneous microwave coagulation therapy for small hepatocellular carcinoma. Cancer 74: 817-825, 1994.

4. Seki T, Wakabayashi M, Nakagawa T, et al: Percutaneous microwave coagulation therapy for patients with small hepatocellular carcinoma: comparison with percutaneous ethanol injection therapy. Cancer 85: 1694-1702, 1999.

5. Murakami R, Yoshimatsu S, Yamashita Y, Matsukawa T, Takahashi M and Sagara K: Treatment of hepatocellular carcinoma: Value of percutaneous microwave coagulation. Am J Roentgenol 164: 1159-1164, 1995.

6. Rossi S, Di Stasi M, Buscarini E, et al: Percutaneous RF interstitial thermal ablation in the treatment of hepatic cancer. Am J Roentgenol 167: 759-768, 1996.

7. Livraghi T, Goldberg SN, Lazzaroni S, Meloni F, Solbiati L and Gazelle GS: Small hepatocellular carcinoma: treatment with radio-frequency ablation versus ethanol injection. Radiology 210: 655-661, 1999

8. Shiina S, Teratani T, Obi S, et al: A randomized controlled trial of radiofrequency ablation with ethanol injection for small hepatocellular carcinoma. Gastroenterology 129: 122-130, 2005.

9. Ishii H, Okada S, Nose H, et al: Local recurrence of hepatocellular carcinoma after percutaneous ethanol injection. Cancer 77 : 1792-1796, 1996.

10. Chopra S, Dodd GD, Chintapalli KN, Leyendecker JR, Karahan OI and Rhim H: Tumor recurrence after radiofrequency thermal ablation of hepatic tumors: Spectrum of findings on dual-phase contrast-enhanced CT. Am J Roentgenol 177: 381-387, 2001.

11. Hori T, Nagata K, Hasuike S, et al: Risk factors for the local recurrence of hepatocellular carcinoma after a single session of percutaneous radiofrequency ablation. J Gastroenterol 38: 977-981, 2003.

12. Okusaka T, Okada S, Ueno H, et al: Satellite lesions in patients with small hepatocellular carcinoma with reference to clinicopathologic features. Cancer 95: 1931-1937, 2002.

13. Maeda T, Takenaka K, Taguchi K, et al: Small hepatocellular carcinoma with minute satellite nodules. Hepatogastroenterology 47: 1063-1066, 2000.

14. Sasaki A, Kai S, Iwashita Y, Hirano S, Ohta M and Kitano S: Microsatellite distribution and indication for locoregional therapy in small hepatocellular carcinoma. Cancer 103: 299-306, 2005.

15. Livraghi T, Solbiati L, Meloni F, Gazelle GS, Halpern EF and Goldberg SN: Treatment of focal liver tumors with percutaneous radio-frequency ablation: complications encountered in multicenter study. Radiology 226: 441-451, 2003.

16. Curley SA, Marra P, Beaty K, et al: Early and late complications after radiofrequency ablation of malignant liver tumors in 608 patients. Ann Surg 239: 450-458, 2004.

17. Baere T, Risse O, Knoch V, et al: Adverse events during radiofrequency treatment of 582 hepatic tumors. Am J Roentgenol 181: 695-700, 2003.

18. Mulier S, Mulier P, Ni Y, et al: Complications of radiofrequency coagulation of liver tumors. Br J Surg 89: 1206-1222, 2002.

19. Lai ECS, You KT, Ng IOL and Shek TWH: The pathological basis of resection margin for hepatocellular carcinoma. World J Surg 17: 786-791, 1993.

20. Yoshida Y, Kanematsu T, Matsumata T, Takenaka K and Sugimachi K: Surgical margin and recurrence after resection of hepatocellular carcinoma in patients with cirrhosis. Further evaluation of limited hepatic resection. Ann Surg 209: 297-301, 1989.

21. Masutani S, Sasaki Y, Imaoka S, et al: The prognostic significance of surgical margin in liver resection of patients with hepatocellular carcinoma. Arch Surg 129: 1025-1030, 1994. 
22. Shi M, Guo RP, Lin XJ, et al: Partial hepatectomy with wide versus narrow resection margin for solitary hepatocellular carcinoma. A Prospective Randomized Trial. Ann Surg 245: 36-43, 2007.

23. Seki S, Sakaguchi H, Iwai S, et al: Five-year survival of patients with hepatocellular carcinoma treated with laparoscopic microwave coagulation therapy. Endoscopy 37: 1220-1225, 2005.

24. Raut CP, Izzo F, Marra P, et al: Significant long-term survival after radiofrequency ablation of unresectable hepatocellular carcinoma in patients with cirrhosis. Ann Surg Oncol 12: 616-628, 2005.
25. Soyer P, Bluemke DA, Hruban RH, Sitzmann JV and Fishman EK: Primary malignant neoplasms of the liver: detection with helical ct during arterial portography. Radiology 192: 389-392, 1994

26. Merine D, Takayasu K and Wakao F: Detection of hepatocellular carcinoma: comparison of CT during arterial portography with CT after intraarterial injection of iodized oil. Radiology 175: 707-710, 1990. 